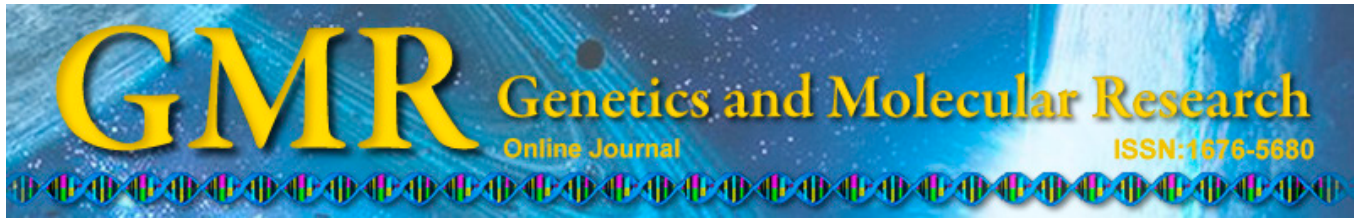

\title{
Role of APN and TNF- $\alpha$ in type 2 diabetes mellitus complicated by nonalcoholic fatty liver disease
}

\author{
X. Lin ${ }^{1,2}$, Z. Zhang', J.M. Chen ${ }^{2}$, Y.Y. Xu' ${ }^{2}$, H.R. Ye ${ }^{2}$, J. Cui ${ }^{2}$, Y. Fang ${ }^{2}$, \\ Y. Jin' ${ }^{2}$ D.R. Zhu' ${ }^{2}$ and L. Yuan ${ }^{1}$ \\ ${ }^{1}$ Department of Endocrinology, Union Hospital, Tongji Medical College, \\ Huazhong University of Science and Technology, Wuhan, China \\ ${ }^{2}$ Department of Endocrinology, \\ China Resource and WISCO General Hospital, Wuhan, China
}

Corresponding author: L. Yuan

E-mail: yuanlidoc@126.com

Genet. Mol. Res. 14 (2): 2940-2946 (2015)

Received May 20, 2014

Accepted November 27, 2014

Published April 10, 2015

DOI http://dx.doi.org/10.4238/2015.April.10.1

\begin{abstract}
Non-alcoholic fatty liver disease (NAFLD) is a chronic liver disease caused by non-excessive alcohol consumption and is the most common cause of elevated levels of serum liver enzymes. We examined changes in adiponectin (APN) and tumor necrosis factor- $\alpha$ (TNF- $\alpha$ ) in type 2 diabetes mellitus (T2DM) complicated by NAFLD and their relationships with insulin resistance (IR). Forty-two T2DM, 39 NAFLD, and 45 T2DM complicated with NAFLD (complicated group) patients were enrolled in this study. Body mass index, fasting blood plasma glucose (FPG), fasting insulin, triglyceride (TG), alanine aminotransferase, gamma-glutamyl transpeptidase, APN, TNF- $\alpha$, and homeostasis model of assessment (HOMA)-IR were determined. The degree of fatty liver was graded according to liver/spleen computed tomography ratio and intrahepatic vessel manifestations. Compared with the T2DM and NAFLD groups, fasting blood plasma glucose, alanine aminotransferase, gamma-glutamyl transpeptidase, TG,
\end{abstract}


TNF- $\alpha$, and HOMA-IR in the complicated group were significantly increased, while APN was significantly reduced. Body mass index in the complicated group was significantly higher than in the T2DM group. The complicated group was prone to severe fatty liver compared with the NAFLD group. APN was negatively correlated with body mass index, fasting blood plasma glucose, TG, TNF- $\alpha$, and HOMA-IR. TNF- $\alpha$ was negatively correlated with APN, but positively correlated with FPG, fasting insulin, TG, and HOMA-IR. The complicated group had clear IR. A more severe degree of fatty liver was associated with higher HOMA-IR and TNF- $\alpha$ and lower APN. APN was an important factor for antagonizing inflammation and mitigating IR.

Key words: Insulin resistance; Nonalcoholic fatty liver disease; Adiponectin; Tumor necrosis factor- $\alpha$; Type 2 diabetes mellitus

\section{INTRODUCTION}

Non-alcoholic fatty liver disease (NAFLD) is a chronic liver disease caused by nonexcessive alcohol consumption and is the most common cause of elevated levels of serum liver enzymes (Hanley et al., 2004). It has been reported that abnormal liver functions in $70 \%$ of diabetic patients are caused by a concurrent fatty liver (Li et al., 2008). Clinically, type 2 diabetes mellitus (T2DM) complicated with NAFLD is more common. A number of studies have suggested that insulin resistance (IR) is not only a characteristic of T2DM occurrence but also the basis of development of NAFLD. IR is a chronic subclinical inflammatory process (Shoelson et al., 2006) that is closely related to adiponectin (APN) and tumor necrosis factor- $\alpha$ (TNF- $\alpha$ ), which are effective indicators of hepatic fatty infiltration (Zhu et al., 2008). In this study, serum APN and TNF- $\alpha$ levels of T2DM patients complicated with NAFLD were measured. We examined the relationship between these indicators and their roles in the occurrence of T2DM and NAFLD.

\section{MATERIAL AND METHODS}

\section{Subjects}

A total of 126 clinic-service and hospitalized patients were enrolled in this study, including 42 cases of T2DM (T2DM group; 20 males and 22 females; mean age of $56 \pm 14$ years; mean disease duration of $1.8 \pm 1.4$ years), 39 cases of NAFLD (NAFLD group; 21 males and 18 females; mean age of $52 \pm 13$ years; mean disease duration of $2.2 \pm 1.2$ years), and 45 cases of T2DM complicated with NAFLD (complicated group; 25 males and 20 females; mean age of $59 \pm 16$ years; mean disease duration of $1.9 \pm 1.6$ years); 30 medically healthy workers in our hospital were selected as the normal control (NC) group (NC group; 14 males and 16 females; mean age of $50 \pm 18$ years). The diagnosis of T2DM met the diagnostic criteria of the World Health Organization in 1999, excluding T1DM infection, stress, secondary diabetes, and gestational diabetes. B-ultrasonically diagnosed NAFLD patients met 
the criteria of simple fatty liver and steatohepatitis proposed by the Fatty Liver and Alcoholic Liver Disease Study Group, Liver Branch of the Chinese Medical Association in 2003. Patients with viral hepatitis, autoimmune hepatitis, drug-induced hepatitis, alcoholic fatty liver, and liver cirrhosis were excluded. There were no statistically significant differences in gender, age, and disease duration among groups.

\section{Methods}

The height $(\mathrm{m})$ and weight $(\mathrm{kg})$ of all subjects were measured to calculate the body mass index (BMI) $\left(\mathrm{kg} / \mathrm{m}^{2}\right.$; accuracy, 0.1$)$. Fasting blood plasma glucose (FPG) was detected using the glucose oxidase method, while fasting insulin (Fins) was detected using a magnetic affinity enzyme-linked immunoassay. The homeostasis model of assessment-insulin resistance $($ HOMA-IR $)$ was calculated as follows: $($ HOMA-IR $)=($ FPG x Fins $) / 22.5$. Triglyceride $(T G)$ was detected using the glycerol-3-phosphate-phenol + aminophenazone method, and alanine aminotransferase (ALT) and gamma-glutamyl transferase (GGT) were detected using a micromethod. Serum APN and TNF- $\alpha$ were measured using an enzyme-linked immunoassay according to manufacturer instructions. Computed tomography (CT) detection was performed in all patients. According to the liver/spleen CT ratio and intrahepatic vessel manifestations, the severity of fatty liver was graded as follows: liver/spleen CT ratio $\leq 1.0$, mild; liver/spleen $\mathrm{CT}$ ratio $\leq 0.7$ with unclear intrahepatic vessels, moderate; liver/spleen $\mathrm{CT}$ ratio $\leq 0.5$ with unclear intrahepatic vessels, severe.

\section{Statistical analysis}

Statistical analysis was performed using the SPSS 11.0 statistical software (SPSS, Inc., Chicago, IL, USA). The measurement data are reported as means $\pm \mathrm{SD}$. Intergroup comparison was performed using analysis of variance, and parameter comparison was performed using correlation analysis, multivariate gradual regression analysis, and logistic gradual regression analysis. Enumeration data were analyzed using the chi-squared test.

\section{RESULTS}

\section{Clinical data}

As shown in Table 1, compared with the NC group, BMI, GGT, TG, TNF- $\alpha$, and HOMA-IR in the T2DM, NAFLD, and complicated groups were significantly higher $(\mathrm{P}<$ 0.05 and $\mathrm{P}<0.01)$, while APN was significantly lower $(\mathrm{P}<0.05$ and $\mathrm{P}<0.01)$. FPG, ALT, GGT, TG, TNF- $\alpha$, and HOMA-IR in the complicated group were significantly higher than in the T2DM and NAFLD groups $(\mathrm{P}<0.05$ and $\mathrm{P}<0.01)$, while APN in the complicated group was significantly decreased $(\mathrm{P}<0.05$ and $\mathrm{P}<0.01)$. BMI in the complicated group was significantly higher than in the T2DM group $(\mathrm{P}<0.01)$, with no significant difference with the NAFLD group $(\mathrm{P}>0.05)$. BMI, ALT, GGT, TG, and APN in the NAFLD group were significantly increased compared with the T2DM group $(\mathrm{P}<0.05$ and $\mathrm{P}<0.01)$, while FPG, Fins, TNF- $\alpha$, and HOMA-IR in the NAFLD group were significantly reduced $(\mathrm{P}<0.05$ and $\mathrm{P}<0.01)$. 


\begin{tabular}{|c|c|c|c|c|}
\hline Item & $\mathrm{NC}(30)$ & T2DM (42) & NAFLD (39) & Complicated (45) \\
\hline BMI $\left(\mathrm{kg} / \mathrm{m}^{2}\right)$ & $21.43 \pm 2.12^{*}$ & $22.49 \pm 2.23^{*}$ & $25.25 \pm 2.93 * * \Delta \Delta$ & $25.71 \pm 2.83^{* * \Delta \Delta}$ \\
\hline $\mathrm{FPG}(\mathrm{mM})$ & $5.12 \pm 0.68$ & $9.08 \pm 0.54 * *$ & $5.28 \pm 074^{\Delta \Delta}$ & $10.03 \pm 0.72 * * \Delta \Delta \$ \S$ \\
\hline Fins $(\mathrm{mU} / \mathrm{L})$ & $8.25 \pm 2.12$ & $11.28 \pm 4.38 * *$ & $9.46 \pm 3.32^{\Delta}$ & $12.63 \pm 4.72 * * \S \S$ \\
\hline $\operatorname{ALT}(\mathrm{U} / \mathrm{L})$ & $22.40 \pm 5.24$ & $24.35 \pm 6.13$ & $48.95 \pm 10.25^{* * \Delta \Delta}$ & $54.74 \pm 15.04 * * \Delta \Delta \S$ \\
\hline GGT (U/L) & $26.43 \pm 13.4$ & $35 \pm 11.6^{* *}$ & $54.2 \pm 11.2^{* * \Delta \Delta}$ & $61.0 \pm 18.5^{* * \Delta \Delta \S}$ \\
\hline $\mathrm{TG}(\mathrm{mM})$ & $1.21 \pm 0.45$ & $1.42 \pm 0.42 *$ & $3.34 \pm 1.35^{* * \Delta \Delta}$ & $4.35 \pm 2.85^{* * \Delta \Delta \S}$ \\
\hline $\mathrm{APN}(\mu \mathrm{g} / \mathrm{mL})$ & $6.08 \pm 1.32$ & $4.55 \pm 1.35^{* *}$ & $5.18 \pm 1.42 * * \Delta$ & $3.15 \pm 2.62 * * \Delta \S \S$ \\
\hline TNF- $\alpha(\mathrm{pg} / \mathrm{mL})$ & $3.46 \pm 0.58$ & $5.49 \pm 1.48 * *$ & $4.92 \pm 1.02 * * \Delta$ & $6.82 .43 \pm 2.97 * * \Delta \S \S$ \\
\hline HOMA-IR & $1.78 \pm 0.58$ & $4.45 \pm 1.35^{* *}$ & $2.22 \pm 1.01 * \Delta \Delta$ & $5.65 \pm 2.14^{* * \Delta \Delta \S \S}$ \\
\hline
\end{tabular}

${ }^{*} \mathrm{P}<0.05$ and ${ }^{* *} \mathrm{P}<0.01$ compared with the $\mathrm{NC}$ group; ${ }^{\circ} \mathrm{P}<0.05$ and ${ }^{\Delta \Delta} \mathrm{P}<0.01$ compared with the T2DM group; ${ }^{\S} \mathrm{P}<0.05$ and ${ }^{\$} \$ \mathrm{P}<0.01$ compared with the NAFLD group. $\mathrm{BMI}=$ body mass index; $\mathrm{FPG}=$ fasting blood plasma glucose; Fins = fasting insulin; ALT = alanine aminotransferase; GGT = gamma-glutamyl transferase; $\mathrm{TG}=$ triglycerides; $\mathrm{APN}=$ adiponectin; TNF- $\alpha=$ tumor necrosis factor-alpha; HOMA-IR = homeostasis model of assessment-insulin resistance; $\mathrm{NC}=$ normal control; $\mathrm{T} 2 \mathrm{DM}=$ type 2 diabetes mellitus; NAFLD = nonalcoholic fatty liver disease.

\section{Imaging results}

The imaging degrees of fatty liver patients are shown in Table 2 . There were significant differences in cases with mild, moderate, and severe fatty liver between the NAFLD and complicated groups $(\mathrm{P}<0.05)$. Liver/spleen CT ratio, ALT, TG, HOMA-IR, APN, and TNF- $\alpha$ levels in fatty liver patients with different imaging degrees are shown in Table 3. With the change in fatty liver degree from mild to severe, the liver/spleen CT ratio was significantly decreased $(\mathrm{P}<0.01)$. Compared with the mild group, ALT, TG, HOMA-IR, and TNF- $\alpha$ in the moderate and severe fatty liver patient groups were significantly increased $(\mathrm{P}<0.05$ and $\mathrm{P}$ $<0.01$ ), while APN was significantly decreased $(\mathrm{P}<0.05$ and $\mathrm{P}<0.01)$. Compared with the moderate group, ALT, HOMA-IR, and TNF- $\alpha$ in the severe group were significantly increased $(\mathrm{P}<0.05$ and $\mathrm{P}<0.01)$, while APN was significantly decreased $(\mathrm{P}<0.05)$, with no significant difference in TG $(\mathrm{P}>0.05)$.

Table 2. Imaging comparison between NAFLD and complicated groups.

\begin{tabular}{lccc}
\hline Item & Mild (cases) & Moderate (cases) & Severe (cases) \\
\hline NAFLD (39) & 22 & 11 & 6 \\
Complicated (45) & $13^{*}$ & $24^{*}$ & $8^{*}$ \\
\hline
\end{tabular}

$* \mathrm{P}<0.05$ compared with NAFLD group.

Table 3. Comparison among fatty liver patients with different imaging degrees.

\begin{tabular}{lcccccc}
\hline Group & Liver/spleen CT ratio & \multicolumn{1}{c}{ ALT } & \multicolumn{1}{c}{ TG } & HOMA-IR & APN & TNF- $\alpha$ \\
\hline Mild (35) & $0.87 \pm 0.09$ & $38.65 \pm 9.54$ & $2.22 \pm 1.29$ & $2.15 \pm 0.99$ & $5.38 \pm 1.25$ & $4.75 \pm 0.96$ \\
Moderate (35) & $0.67 \pm 0.09^{* *}$ & $49.02 \pm 12.88^{* *}$ & $4.18 \pm 1.78^{* *}$ & $4.69 \pm 1.32^{* *}$ & $4.15 \pm 1.44^{* *}$ & $5.48 \pm 1.67 *$ \\
Severe (14) & $0.46 \pm 0.05^{* * \Delta \Delta}$ & $63.71 \pm 14.75^{* * \Delta \Delta}$ & $5.24 \pm 2.08^{* *}$ & $5.76 \pm 2.26^{* * \Delta}$ & $3.02 \pm 2.35^{* * \Delta}$ & $7.11 \pm 2.45^{* * \Delta}$ \\
\hline
\end{tabular}

$* \mathrm{P}<0.05$ and $* * \mathrm{P}<0.01$ compared with mild group; ${ }^{\circ} \mathrm{P}<0.05$ and ${ }^{\Delta \Delta} \mathrm{P}<0.01$ compared with moderate group. 


\section{Correlation analysis and regression analysis results}

Age, disease duration, BMI, FPG, Fins, ALT, GGT, TG, APN, TNF- $\alpha$, and HOMA-IR were used as the independent variables, and fatty liver degree was used as the dependent variable for logistic gradual regression analysis. The results showed that BMI, TG, and HOMA-IR were the major risk factors of NAFLD occurrence.

APN was used as the dependent variable for simple correlation analysis with age, duration, BMI, FPG, Fins, ALT, GGT, TG, TNF- $\alpha$, HOMA-IR, and liver/spleen CT ratio, respectively. The results revealed that APN was negatively correlated with BMI $(r=-0.588)$, FPG $(r=-0.286)$, TG $(r=-0.236)$, TNF- $\alpha(r=-0.338)$, and HOMA-IR $(r=-0.213)$, while the correlations with other variables were not significant. The gradual regression analysis revealed that the selected variables were BMI and TNF- $\alpha$.

TNF- $\alpha$ was also used as the dependent variable for simple correlation analysis with age, duration, BMI, FPG, Fins, ALT, GGT, TG, APN, and HOMA-IR. The results revealed that TNF- $\alpha$ was negatively correlated with APN $(r=-0.388)$, but positively correlated with FPG $(r=0.217)$, Fins $(r=0.145)$, TG $(r=0.314)$, and HOMA-IR $(r=0.292)$. The correlations between TNF- $\alpha$ with other variables were not significant. Further gradual regression analysis revealed that the variables selected were APN and TG.

\section{DISCUSSION}

In conjunction with social progress and economic prosperity, as well as changes in diet and lifestyle, the rates of T2DM and NAFLD are increasing. The prevalence of NAFLD in the general population is approximately $10-24 \%$, but may reach $75 \%$ in the diabetic population (Zhao et al., 2008a). Liver biopsy is the gold standard for diagnosing fatty liver, but because it is an invasive examination, patient compliance is poor, limiting its clinical application. The sensitivity of ultrasonic B-scanning for diagnosing hepatic steatosis is $100 \%$ and the specificity is $80 \%$ (Graif et al., 2000), showing a higher compliance rate for pathological diagnosis. However, because of the complexity, diversity, and non-homogeneity in liver ultrasound images, individual differences are high, and thus this method cannot be used as an accurate quantitative indicator. $\mathrm{CT}$ is highly sensitive in detecting density differences. There are significant differences in $\mathrm{CT}$ values in the normal human liver, but the values are higher than those in the spleen (the CT value is relatively constant). The CT value of the fatty liver is often lower than in the normal spleen tissue, and CT values are negatively correlated with TG deposition. The determination of the severity grading of fatty liver is generally more accepted than for spleen CT values (Liu and Gao, 2002). Therefore, we used the B-ultrasonic method to diagnose fatty liver and used CT scanning for severity grading of the fatty liver. The results showed that the occurrence of NAFLD was unrelated to gender, which is consistent with studies examining large samples (Jie et al., 2009). The main risk factors of the prevalence were BMI, TG, and HOMA-IR. The duration of disease in patients uncomplicated with T2DM was shorter than in those complicated with T2DM.

IR and hyperinsulinemia are considered to be the main features of T2DM. The "second-hit" theory is the more common pathogenesis of NAFLD. The initial hit involves the IR provoking the accumulation of liver TG until steatosis. The second hit refers to the lipid peroxidation damage caused by the increased oxidation of inflammatory factors, free fatty acids, and TGs. Studies have shown that IR is not only involved in the initial hit but also directly 
involved in the second hit, playing an important role in the development of NAFLD (Zhao et al., 2008b). This study also showed that T2DM and NAFLD patients all had IR, while T2DM patients complicated with NAFLD had a higher degree of IR, which is consistent with the results of a previous study (Xu, 2008). Logistic regression analysis showed that TG and HOMAIR were independent predictors of NAFLD. The insulin sensitizer pioglitazone was reported to improve the histological lesions and liver enzyme indicators in NAFLD patients, NAFLD patients complicated with impaired glucose tolerance, and T2DM patients (Marchesini et al., 2001; Belfort et al., 2006), and that slow sustained weight loss can improve IR and steatosis in NAFLD patients.

APN is a bioactive protein secreted by fat cells that regulates glucose and lipid metabolism, improves the sensitivity to insulin, lowers blood sugar, and improves IR; it also may enhance the expression of lipoprotein lipase, therefore reducing TG synthesis in liver cells and alleviating the steatosis of hepatic fatty cells (Zhong et al., 2008) to combat the inflammation reaction (Okamoto et al., 2006). Numerous studies have found that low APN is characteristic of fatty liver independently from IR and TNF- $\alpha$ (Hui et al., 2004; Musso et al., 2005a), the level of which was negatively correlated with liver steatosis, inflammatory necrosis, and fibrosis (Musso et al., 2005b). In T2DM, particularly in obese T2DM, plasma APN level also decreased significantly, which was negatively correlated with IR and hyperinsulinemia (Weyer et al., 2001). In this study, the serum APN levels of the 3 groups decreased, and was the most significant in T2DM patients complicated with NAFLD, followed by the T2DM group and the NAFLD group. The CT results indicated that the T2DM patients complicated with NAFLD were more likely to suffer from moderate and severe fatty liver compared to NAFLD patients. A more severe degree of fatty liver was associated with higher TG and ALT, as well as higher IR and lower APN. This indicated that with the accumulation of TG in the liver, the inflammation of liver cells was more severe. IR and APN were involved in this inflammatory response. This is consistent with the results of Shoelson et al. (2006). Correlation analysis and gradual regression analysis showed that APN was negatively correlated with BMI, FPG, TG, TNF- $\alpha$, and HOMA-IR, among which the relationship between BMI and TNF- $\alpha$ was the closest.

Inflammation is a key mechanism in the process during which the simple fatty liver develops into steatohepatitis and cirrhosis. TNF- $\alpha$ is a common inflammatory factor that can reduce the levels of the muscle cell glucose transporter $4 \mathrm{mRNA}$ and its protein product (Stephens and Pekala, 1991), inhibit the autophosphorylation of insulin receptors (Storz et al., 1998) and the activation of the second messenger (Miura et al., 1999), and reduce insulindependent glucose uptake; it can also inhibit lipogenesis and stimulate lipolysis, increasing the levels of free fatty acids and affecting lipid metabolism. Studies have found that IR level is positively correlated with fat cell-secreted serum TNF- $\alpha$ level (Pring et al., 1997). APN inhibits TNF- $\alpha$ generation and TNF- $\alpha$ mRNA expression, improving the IR state (Weyer et al., 2001). In this study, TNF- $\alpha$ levels in T2DM patients complicated with NAFLD were significantly higher than in T2DM patients and NAFLD patients. A more severe fatty liver status was associated with higher levels of serum ALT and TNF- $\alpha$. Correlation analysis and gradual regression analysis showed that TNF- $\alpha$ was positively correlated with FPG, Fins, TG, and HOMA-IR and negatively correlated with APN, among which APN and TG were primary factors. Lin (2008) examined diet and exercise interference in T2DM patients, associated with oral administration of metformin, the combination of glitazones/insulin to decrease blood sugar, and fenofibrate to reduce lipids for 1 year. The results revealed that FPG, Fins, HOMAIR, TNF- $\alpha$, and C-reactive protein were significantly reduced, while APN was significantly 
increased. The comparison before and after therapy showed a statistically significant difference, suggesting that inflammation and the low level of APN can be improved through blood glucose and lipid control.

In conclusion, patients with T2DM complicated with NAFLD have obvious IR. A more severe degree of fatty liver was associated with higher HOMA-IR and TNF- $\alpha$ levels and with lower APN levels. APN is an important factor that antagonizes inflammation and ameliorates IR.

\section{REFERENCES}

Belfort R, Harrison SA, Brown K, Darland C, et al. (2006). A placebo-controlled trial of pioglitazone if subjects with nonalcoholic steatohepatitis. N. Engl. J. Med. 355: 2297-2307.

Graif M, Yanuka M, Baraz M, Blank A, et al. (2000). Quantitative estimation of attenuation in ultrasound video images: correlation with histology in diffuse liver disease. Invest. Radiol. 35: 319-324.

Hanley AJ, Williams K, Fests A, Wagenknecht LE, et al. (2004). Elevations in markers of liver injury and risk of type 2 diabetes: the insulin resistance atherosclerosis study. Diabetes 53: 2623-2632.

Hui JM, Hodge A, Farrell GC, Kench JG, et al. (2004). Beyond insulin resistance in NASH: TNF-alpha or adiponectin? Hepatology 40: 46-54.

Jie YS, Xu QH and Zhang K (2009). Screening of prevalence of fatty liver in Guangzhou 4365 B ultrasound health examination cases. New Med. 40: 231-232.

Li XH, Wang Y and Dong LP (2008). The changes of insulin, blood lipid and APN in T2DM patients associated with NAFLD. Shandong Med. 48: 86-87.

Lin HW (2008). The correlation study of APN and inflammatory factors in T2DM patients. Modern Hosp. 8: 11-13.

Liu J and Gao X (2002). The research development of fat liver and diabetes. Med. Summary 8: 657-659.

Marchesini G, Brizi M, Bianchi G, Tomassetti S, et al. (2001). Nonalcoholic fatty liver disease: a feature of the metabolic syndrome. Diabetes 50: 1844-1850.

Miura A, Ishizuka T, Kanoh Y, Ishizawa M, et al. (1999). Effect of tumor necrosis factor-alpha on insulin signal transduction in rat adipocytes: relation to PKC beta and zeta translocation. Biochem. Biophys. Acta 1449: 227-238.

Musso G, Gambino R, Biroli G, Carello M, et al. (2005a). Hypoadiponectinemia predicts the severity of hepatic fibrosis and pancreatic Beta-cell dysfunction in nondiabetic nonobese patients with nonalcoholic steatohepatitis. Am. J. Gastroenterol 100: 2438-2446.

Musso G, Gambino R and Durazzo M (2005b). Adipokines in NASH: postprandial lipid metabolism as a link between adiponectin and liver disease. Hepatology 42: 1175-1183.

Okamoto Y, Kihara S, Funahashi T, Matsuzawa Y, et al. (2006). Adiponectin: a key adipocytokine in metabolic syndrome. Clin. Sci. 110: 267-278.

Pring JB, Niesler CU, Winterford CM, Bright NA, et al. (1997). Tumor necrosis factor-alpha induces apoptosis of human adipose cells. Diabetes 46: 1939-1944.

Shoelson SE, Lee J and Goldfine AB (2006). Inflammation and insulin resistance. J. Clin. Invest. 116: 1793-1801.

Stephens JM and Pekala PH (1991). Transcriptional repression of the GLUT4 and C/EBP genes in 3T3-L1 adipocytes by tumor necrosis factor-alpha. J. Biol. Chem. 266: 21839-21845.

Storz P, Doppler H, Wernig A, Pfizenmaier K, et al. (1998). TNF inhibits insulin induced STAT5 activation in differentiated mouse muscle cells pmi28. FEBS Lett. 440: 41-45.

Weyer C, Funahashi T, Tanaka S, Hotta K, et al. (2001). Hypoadiponectinemia in obesity and type 2 diabetes: close association with insulin resistance and hyperinsulinemia. J. Clin. Endocrinol. Metab. 86: 1930-1935.

Xu XM (2008). Clinical analysis of T2DM patients associated with NAFLD. J. Pract. Diabetes 4: 23-24.

Zhao L, Shrestha D, Zhang X, Wang Y, et al. (2008a). The retrospective analysis of T2DM patients associate with NAFLD. Chin. J. Diabetes 16: 535-536.

Zhao WH, Han DW, Miao YC, Zhang XY, et al. (2008b). The roles of insulin resistance in the occurrence of NAFLD. J. Shanxi Med. Univ. 39: 500-503.

Zhong XL, Zhong DF, Yang Li, Ai ZL, et al. (2008). The expression of Lipoprotein Lipase in the steatosis of liver cells and the interventional role of APN. Chongqing Med. J. 37: 2199-2202.

Zhu CY, Zhou H, Han Y, Ling LQ, et al. (2008). The application of APN, TNF $\alpha$ and ferroprotein in the Detection of T2DM patients associated with FLD. Nuclear Technol. 31: 356-359. 